\title{
Case Report and Review of the Literature
}

\section{Rectovaginal Fistula on Migration of an Intrauterine Device: A Case Report and Literature Review}

\author{
Sophie Schoenen ${ }^{I^{*}}$, Pascal De Leeuw ${ }^{2}$, Vlad-Adrian Alexandrescu ${ }^{1}$ and Christian Ngongang ${ }^{1}$ \\ ${ }^{I}$ Department of General, Abdominal, Thoracic and Vascular Surgery, Princess Paola Hospital, Marche-en-Famenne, Belgium \\ ${ }^{2}$ Department of Gastroenterology, Princess Paola Hospital, Marche-en-Famenne, Belgium
}

\begin{tabular}{l} 
A R T I C L E I N F O \\
\hline Article history: \\
Received: 5 March, 2021 \\
Accepted: 19 March, 2021 \\
Published: 22 April, 2021 \\
\hline Keywords: \\
Intrauterine device \\
migration \\
rectovaginal fistula \\
laparoscopic surgery
\end{tabular}

\begin{abstract}
A B S T R A C T
Intrauterine devices (IUDs) are effective methods of contraception widely used worldwide because of their efficacy, low cost, and reversibility. Although overall well-tolerated, they may, however, cause uterine perforation, a rare but serious complication that can endanger adjacent abdominal organs. We report the case of a 50-year-old woman who consulted a gastroenterologist three years ago for the detection of blood in her feces on a screening test $\left(\right.$ Hémoccult $\left.^{\circ}\right)$. Colonoscopy and abdominopelvic imaging discovered a displaced IUD embedded in the recto-sigmoidal wall. A wait-and-see approach was proposed at that time because of the absence of symptoms. Three years later, she developed abdominal and pelvic pain. On a second laparoscopic surgery procedure, we removed the IUD. Intraoperatively, a rectovaginal fistula was also discovered and sutured in different anatomical planes. Based on a comprehensive review of the literature, the management of this case is confronted with guidelines available for this rare condition.
\end{abstract}

\section{Introduction}

Intrauterine devices (IUDs) are widely used methods of contraception because of their effectiveness and low cost. However, they are associated with rare, but potentially serious complications, including migration through the myometrium, which can lead to digestive fistulas. Here we report an illustrative case that enhanced several management problems.

\section{Case Presentation}

In 2018, a 50-year-old patient consulted the gastroenterology department because of a positive fecal haemoglobin detection test (Hémoccult ${ }^{\circ}$ ) performed as part of a family screening due to her father's colorectal polyps. She was not complaining about transit disorders, rectal bleeding, or melena. Gynaecologically, she has been suffering from adenomyosis responsible for menorrhagia controlled by a macroprogestin treatment $\left(\right.$ Luteny $1^{\circ}$ ) for several years. She is mother of three children: two vaginal deliveries and one cesarean section.
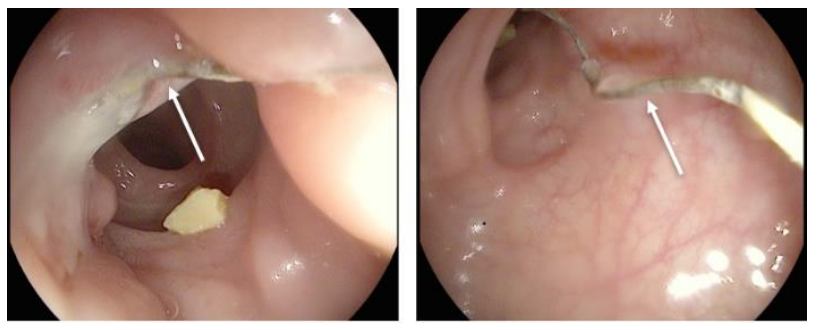

Figure 1: Colonoscopic image showing a polypoid sigmoid mass with a "suture thread" protruding in its center (arrows).

At colonoscopy, a polypoid sigmoid mass with an inflammatory appearance was detected with a "suture thread" protruding in its center, about $15 \mathrm{~cm}$ away from the anal margin (Figure 1). Biopsy of this mass showed the presence of granulation tissue with no carcinomatous infiltration. Following these findings, pelvic magnetic resonance imaging (MRI) was performed. This exam revealed an IUD in an ectopic position: one horizontal branch of the ' $\mathrm{T}$ ' appeared to sit within the mesorectal fat, the other showed to be in contact with the posterior vaginal recess. The vertical branch reached the recto-sigmoidal junction,

\footnotetext{
${ }^{*}$ Correspondence to: Sophie Schoenen, M.D., Department of General, Abdominal, Thoracic and Vascular Surgery, Princess Paola Hospital, Vivalia, Marche-enFamenne, Belgium; E-mail: sophieschoenen@gmail.com
} 
without demonstrated parietal transfixion (Figure 2). The presence of an $11 \mathrm{~mm}$ sigmoid polypoid formation is also confirmed at that moment; it was not clear whether this was a true polyp or a foreign body reaction.

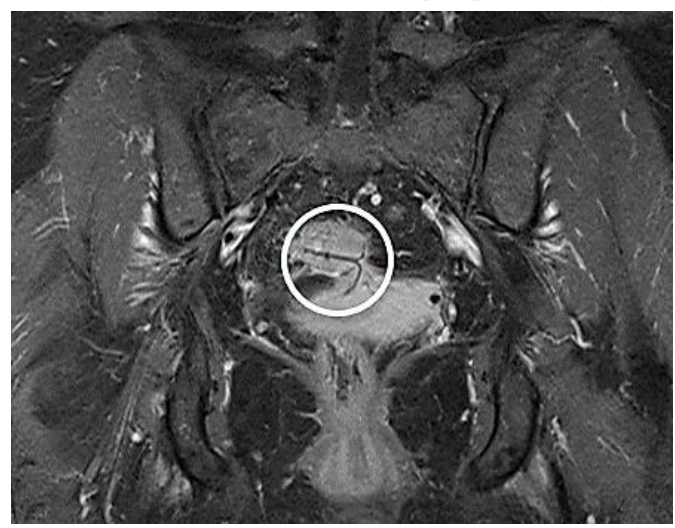

Figure 2: MRI (coronal view) reveals a displaced IUD (circle) with a branch reaching the wall of the recto-sigmoidal junction.

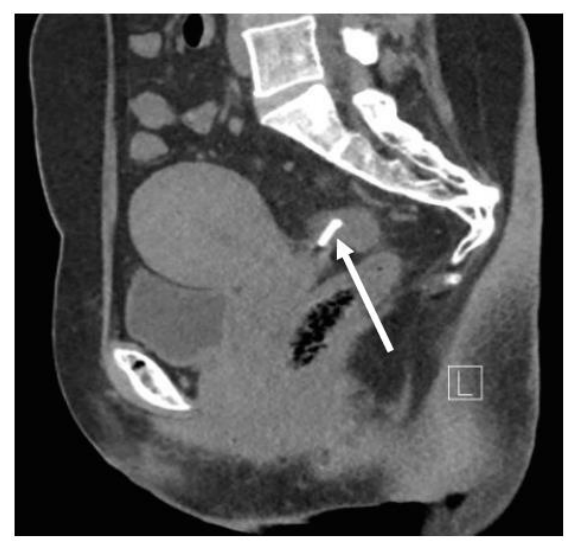

A
A more exhaustive anamnesis revealed that 20 years ago the patient had indeed a levonorgestrel IUD inserted. In the hours following the procedure, she had hemorrhagic vaginal bleeding that lasted for several days. Not visualizing the IUD at the ultrasound examination, her gynaecologist concluded that the IUD had been expelled early and inserted another one, which will be removed, however, two years later, as to allow her third pregnancy to take place. As a matter of fact, an ectopic IUD was discovered during pelvimetry before her last delivery in 2001. Since there were no symptoms, the device was left in place. Similarly, after the 2018 colonoscopy, as the patient remains asymptomatic, a wait-and-see attitude is decided, as surgical exploration would have entailed an eventual risk of colorectal resection with transient ileostomy.

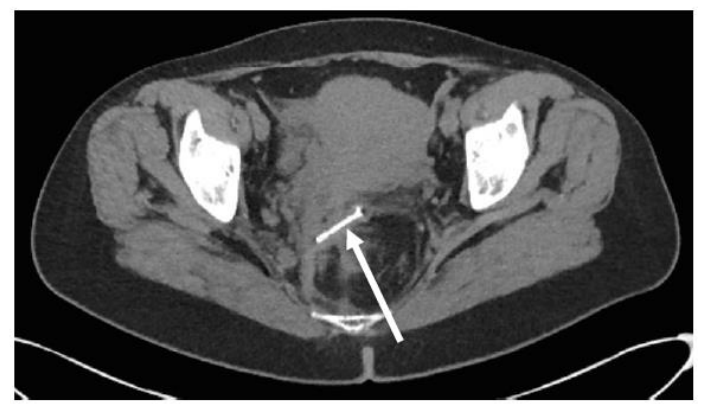

B

Figure 3: CT-scan confirms the IUD (arrow) in ectopic location at the level of the sigmoid wall. A) Sagittal view, B) transversal view.

Two years later, after being "badly shaken during a ride on a fairground attraction", the patient consults for intense pain in the hypogastrium and both iliac fossae. On clinical examination, the abdomen appears sensitive to palpation but without abdominal guarding. An abdominal CT-scan performed in the emergency room confirms the IUD in the ectopic location at the level of the sigmoid wall (Figure 3). A new colonoscopy is performed to judge its exact position and evaluate the possibility of endoscopic removal. The situation is unchanged compared to 2018; a single intra-colic suture thread is visualized.

Hence an exploratory laparoscopy is planned. The patient, however, prefers to delay this surgery for professional reasons; it will not be performed until 5 months later. Laparoscopic abdominal exploration reveals a very inflammatory aspect of the pelvis with a right para-rectal plastron. Perirectal dissection does not detect the IUD. Intraoperative colonoscopy shows a complete disappearance of the suture thread. Taking into account the distal location of the inflammatory bowel (which would impose an ileostomy in case of rectal resection), the operation is not continued. Post-operative plain abdominal X-ray confirms the presence of an IUD still located next to the sacrococcygeal junction.
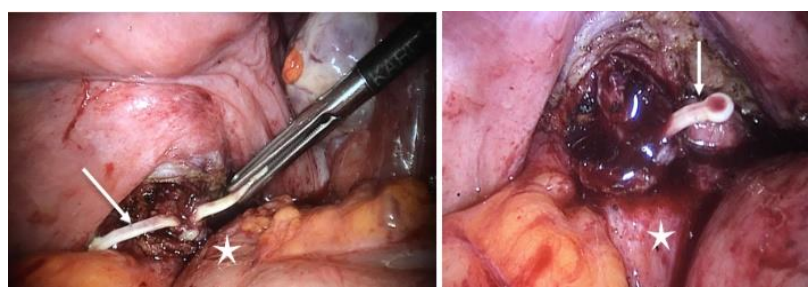

Figure 4: Laparoscopic images showing the IUD (arrow) embedded in the vaginal wall next to the rectum (star).

Due to further persistent pelvic pain, we performed a new exploratory laparoscopy 4 months later, the patient being informed of the potential risk of distal rectal resection and transient ileostomy. Careful bilateral dissection of the latero-rectal and para-uterine peritoneum allows mobilization of the entire mesorectum in Heald's dissection plane. The opening of the rectovaginal septum reveals a chronic abscess with rectovaginal fistulation. Intraoperative rectoscopy confirms the presence of an inflammatory area $12 \mathrm{~cm}$ from the anal margin, without intra-rectal foreign body. This aspect corresponds to the blind orifice of a rectovaginal fistula. Continued dissection evinces the IUD in the vaginal wall, whose opening allows for its easy removal (Figure 4). Rectal injection of isobetadine does not reveal an intra-abdominal leakage. Nevertheless, we prefer to reinforce the rectal wall with a separate 
suture. The vaginal fundus is then reconstructed in two planes. The postoperative follow-up is eventless, and the patient is able to return home on the 3rd post-operative day.

\section{Discussion}

Millions of women use IUDs as a contraceptive method, found more in developing countries $(14.5 \%$ of users versus $7.6 \%$ in developed countries) [1]. Although rare, uterine perforation is a serious complication with an incidence of 0.3 to $2.6 / 1000$, without significant difference between copper and levonorgestrel IUDs [2]. In the majority of cases, the perforation appears primary, i.e., it occurs during the insertion of the device, but it often remains unrecognized [3]. It is for this reason that a transvaginal ultrasound scan is recommended after IUD insertion. Secondary perforations occur later, within 5 years of insertion [3]. Intra-abdominal migration must be suspected when the threads of the device are not visualized with speculum examination during the gynaecological follow-up. An exhaustive exploration by pelvic ultrasound, abdominal X-ray, CT-scan, or pelvic MRI, or even an endoscopic examination (hysteroscopy, colonoscopy, cystoscopy) must then be performed in order to locate the IUD [4]. Spontaneous expulsion should be considered only as a diagnosis of exclusion. The risk factors for uterine perforation are the postpartum period, breastfeeding, uterine malformations, and inexperience of the operator $[5,6]$. The age of the patient does not seem to influence this risk.

The clinical presentation varies from patient to patient and occurs at different delays. It consists of either a total absence of symptoms $(34.5 \%)$, or abdominal pain $(34.5 \%)$, vaginal bleeding, unwanted pregnancy, or infertility [6]. In $15 \%$ of cases, the device reaches the adjacent organs, which may be responsible for fistula, obstruction, and peritonitis. The gastrointestinal tract is most often impacted: the sigmoid detains a higher incidence ( $40.4 \%$ ), followed by the small intestine and the rectum [3]. The management of IUD ectopic migration remains controversial when the patient is asymptomatic. The World Health Organization advocates a proactive approach with immediate removal of the device because of potentially serious complications (digestive or urinary tract damage, adhesion formation) [7]. Exploratory laparoscopy is recommended as a first-line procedure with a success rate of $40 \%$ to $100 \%$, depending on the surgeon's experience [8]. An endoscopic approach (colonoscopy, cystoscopy) can also be proposed in case of migration in the colon or bladder. Some teams, however, advocate a conservative treatment with a wait-and-see attitude aimed at limiting invasive procedures $[9,10]$.

\section{Conclusion}

IUDs are effective and widely used contraceptive methods around the globe. Rare but potentially harmful uterine perforation may appear, which can lead to digestive or urinary fistulas. Since IUD threads are not visible on gynaecological examination, endovaginal ultrasound, abdominal X-ray, or CT-scan, or even colonoscopy/cystoscopy are recommended to precisely locate the device. Surgical removal is the recommended approach to avoid serious long-term complications. Laparoscopic surgery remains the method of first choice.

\section{Conflicts of Interest}

None.

\section{REFERENCES}

1. d'Arcangues C (2007) Worldwide use of intrauterine devices for contraception. Contraception 75: S2-S7. [Crossref]

2. Heinemann K, Reed S, Moehner S, Minh TD (2015) Risk of uterine perforation with levonorgestrel-releasing and copper intrauterine devices in the European Active Surveillance Study on intrauterine devices. Contraception 91: 274-279. [Crossref]

3. Toumi O, Ammar H, Ghdira A, Chhaidar A, Trimech W et al. (2018) Pelvic abscess complicating sigmoid colon perforation by migrating intrauterine device: A case report and review of the literature. Int $J$ Surg Case Rep 42: 60-63. [Crossref]

4. Adiyeke M, Sanci M, Karaca I, Gökçü M, Töz E et al. (2015) Surgical management of intrauterine devices migrated towards intra-abdominal structures: 20-year experience of a tertiary center. Clin Exp Obstet Gynecol 42: 358-360. [Crossref]

5. Kaislasuo J, Suhonen S, Gissler M, Lähteenmäki P, Heikinheimo O (2012) Intrauterine contraception: incidence and factors associated with uterine perforation: a population-based study. Hum Reprod 27: 26582663. [Crossref]

6. Sun X, Xue M, Deng X, Lin Y, Tan Y et al. (2018) Clinical characteristic and intraoperative findings of uterine perforation patients in using of intrauterine devices (IUDs). Gynecol Surg 15: 3. [Crossref]

7. World Health Organization (1987) Mechanism of action, safety and efficacy of intrauterine devices. Report of a WHO Scientific Group. World Health Organ Tech Rep Ser 753: 1-91. [Crossref]

8. Ozgun M, Batukan C, Serin IS, Ozcelik B, Basbug M et al. (2007) Surgical management of intra-abdominal mislocated intrauterine devices. Contraception 75: 96-100. [Crossref]

9. Adoni A, Ben CA (1991) The management of intrauterine devices following uterine perforation. Contraception 43: 77-81. [Crossref]

10. Markovitch O, Klein Z, Gidoni Y, Holzinger M, Beyth Y (2002) Extrauterine mislocated IUD: is surgical removal mandatory? Contraception 66: 105-108. [Crossref] 